\title{
Curie-Weiss-type law for the strain and stress effects on the dielectric response of ferroelectric thin films
}

\author{
N. A. Pertseva) \\ Elektrokeramische Materialen, Institut für Festkörperforschung, Forschungszentrum Jülich, \\ D-52425 Jülich, Germany \\ V. G. Koukhar \\ A. F. Ioffe Physico-Technical Institute, Russian Academy of Sciences, 194021 St. Petersburg, Russia \\ R. Waser and S. Hoffmann \\ Elektrokeramische Materialen, Institut für Festkörperforschung, Forschungszentrum Jülich, D-52425 Jülich, \\ Germany
}

(Received 20 January 2000; accepted for publication 23 August 2000)

\begin{abstract}
Variations of the dielectric properties of ferroelectric thin films with the misfit strain $S_{m}$ in the film/substrate system and the associated biaxial stress $\sigma$ inside the film are analyzed theoretically. Calculations are performed in a mean-field approximation for the dielectric response displayed in a plate-capacitor setup by single crystalline films epitaxially grown on tensile substrates $\left(S_{m}>0\right)$. It is shown that, in the absence of misfit-strain-induced phase transitions, the film dielectric susceptibility $\eta_{33}$ obeys the Curie-Weiss-type law $\eta_{33}\left(S_{m}\right)=\varepsilon_{0} K_{S} /\left(S_{m}-S_{m}^{*}\right), \quad \eta_{33}(\sigma)$ $=\varepsilon_{0} K_{\sigma} /\left(\sigma-\sigma^{*}\right)$. Theoretical predictions are compared with the measured stress dependence of the dielectric response of polycrystalline $\mathrm{Ba}_{0.7} \mathrm{Sr}_{0.3} \mathrm{TiO}_{3}$ films grown on $\mathrm{Pt} / \mathrm{SiO}_{2} / \mathrm{Si}$. The theory explains the observed dielectric behavior and predicts the existence of in-plane polarization state in this film/substrate system. (C) 2000 American Institute of Physics. [S0003-6951(00)03542-7]
\end{abstract}

For many applications of ferroelectric thin films, such as cells for GBit dynamic random access memories, integrated decoupling capacitors, high-frequency transducers, etc., a high electric permittivity is required. ${ }^{1}$ The dielectric properties of a thin film, however, depend not only on the ferroelectric substance itself, but also on the substrate material and other factors. In particular, these properties should be strongly affected by the lattice misfit between the film and substrate. ${ }^{2-4}$

The misfit-strain changes directly manifest itself in the variation of the in-plane lattice parameter, which can be evaluated by the x-ray diffraction. ${ }^{2}$ Even in the same film/ substrate system, the misfit strain $S_{m}$ may differ for films of different thicknesses because of the strain relaxation caused by the formation of misfit dislocations. ${ }^{5}$ Strong changes of $S_{m}$ with increasing film thickness are evident in $\mathrm{BaTiO}_{3} / \mathrm{Pt} / \mathrm{MgO}$ and $\mathrm{BaTiO}_{3} / \mathrm{SrTiO}_{3}$ heterostructures. ${ }^{2,6}$ The misfit strain can also be tuned to a certain extent by bending the substrate by an external load. Such a bending test was recently performed by Shaw et al. for $\mathrm{Ba}_{0.7} \mathrm{Sr}_{0.3} \mathrm{TiO}_{3}$ films grown on platinum coated silicon substrates. ${ }^{7}$ They evaluated the associated change of the in-plane biaxial stress $\sigma$ in the film and attributed the observed variation of the capacitance to the stress effect. During the dielectric measurements, however, the film grown on a thick substrate is under a fixed strain, but not at constant stress. ${ }^{3}$ Besides, in the presence of spontaneous polarization, the stress is not directly related to the strain. ${ }^{2}$ Therefore, the stress effect is not equivalent to the underlying misfit-strain one.

\footnotetext{
${ }^{a)}$ Permanent address: A. F. Ioffe Physico-Technical Institute, Russian Academy of Sciences, 194021 St. Petersburg, Russia; electronic mail: pertsev@domain.ioffe.rssi.ru
}

In this letter, we analyze the strain and stress effects on the dielectric response exhibited by perovskite ferroelectric films in a conventional plate-capacitor setup. For clarity, we consider only ferroelectric overlayers grown on cubic substrates providing positive misfit strains in the heterostructure.

To describe the above effects theoretically, we will calculate the dielectric response of a single crystalline epitaxial film using the thermodynamic theory, which was developed recently. ${ }^{3,4}$ In contrast with Refs. 3 and 4, a specific feature of the film dielectric properties will be reported here. Namely, our concept is to demonstrate that the reciprocal dielectric susceptibility of a ferroelectric thin film may be a linear function of the misfit strain $S_{m}$ (or internal stress $\sigma$ ) even in the presence of a substantial spontaneous polarization $P_{s}$.

Consider a (001)-oriented film grown on a thick (001) substrate above the temperature of ferroelectric phase transition. When the film is in a paraelectric state, the reciprocal dielectric susceptibility $\chi_{33}$ in the film thickness direction is a linear function of the misfit strain: $^{3,4} \quad \chi_{33}=2 a_{1}$ $-4 S_{m} Q_{12} /\left(s_{11}+s_{12}\right)$, where $a_{1}$ is the dielectric stiffness of a bulk crystal, which linearly depends on temperature $T, Q_{12}$ is the electrostrictive constant of paraelectric phase, and $s_{i j}$ are the film elastic compliances at constant polarization. Therefore, to describe the misfit-strain dependence of the dielectric susceptibility $\eta_{33}$, a Curie-Weiss-type law

$$
\eta_{33}\left(S_{m}\right)=\frac{\varepsilon_{0} K_{s}}{S_{m}-S_{m}^{*}},
$$

may be introduced on the analogy of the well-known CurieWeiss law $\eta_{33}(T) \sim(T-\theta)^{-1}$. Here, $K_{S}=-\left(s_{11}\right.$ $\left.+s_{12}\right) /\left(4 \varepsilon_{0} Q_{12}\right)$, and $S_{m}^{*}(T)=a_{1}(T)\left(s_{11}+s_{12}\right) /\left(2 Q_{12}\right)$ is 
the critical misfit strain, at which the paraelectric phase loses its stability against the appearance of the out-of-plane polarization $P_{3}$. Since in the absence of spontaneous polarization the in-plane biaxial stress $\sigma$ equals $\sigma=S_{m} /\left(s_{11}+s_{12}\right)$, the dependence $\eta_{33}(\sigma)$ obeys the relation

$$
\eta_{33}(\sigma)=\frac{\varepsilon_{0} K_{\sigma}}{\sigma-\sigma^{*}}
$$

similar to Eq. (1), with $K_{\sigma}=-\left(4 \varepsilon_{0} Q_{12}\right)^{-1}$ and $\sigma^{*}$ $=a_{1} /\left(2 Q_{12}\right)$. This corresponds to a linear relationship for $\chi_{33}(\sigma)$ discussed by Shaw et al. ${ }^{7}$ However, as follows from the misfit strain-temperature phase diagrams of ferroelectric thin films, ${ }^{3,4}$ Eqs. (1) and (2) are valid only at misfit strains not exceeding the second critical value $S_{m}^{* *}=a_{1}\left(s_{11}\right.$ $\left.+s_{12}\right) /\left(Q_{11}+Q_{12}\right)$, at which the paraelectric state loses its stability against the appearance of the in-plane polarization.

Since the ferroelectric phase transition in thin films is of the second order, just below the transition temperature the sixth-order polarization terms in the free-energy expansion may be neglected. In the resulting $P^{4}$ approximation, the Curie-Weiss-type law [Eqs. (1) and (2)] remains valid, despite the appearance of a nonzero spontaneous polarization $\mathbf{P}_{\mathrm{s}}$. Indeed, the polarization correction to the reciprocal susceptibility $\chi_{33}$ is proportional to $P_{s}^{2}$, which linearly depends on the strain $S_{m}$ in the discussed case. However, the parameters involved in Eqs. (1) and (2) now differ from those characterizing the paraelectric state. For single-domain $\mathrm{BaTiO}_{3}$ (BT) and $\mathrm{PbTiO}_{3}$ (PT) films, where the ferroelectric transition at $S_{m}>0$ results in the formation of the orthorhombic aa phase with in-plane orientation of $\mathbf{P}_{\mathbf{s}},{ }^{3,4}$ we obtain

$$
\begin{aligned}
& S_{m}^{*}=\frac{a_{1}(1-2 r)\left(s_{11}+s_{12}\right)}{2\left[-Q_{12}+r\left(Q_{11}+Q_{12}\right)\right]}, \\
& K_{s}=\frac{s_{11}+s_{12}}{4 \varepsilon_{0}\left[-Q_{12}+r\left(Q_{11}+Q_{12}\right)\right]},
\end{aligned}
$$

where $r=a_{13}^{*} /\left(2 a_{11}^{*}+a_{12}^{*}\right), a_{i j}^{*}$ are the energy-function coefficients introduced in Ref. 3, and Eq. (1) with these parameters is valid at $S_{m}>S_{m}^{* *}$. Since the in-plane stress $\sigma$ in the aa phase equals $\sigma=\left[S_{m}-\left(Q_{11}+Q_{12}\right) P_{s}^{2} / 2\right] /\left(s_{11}+s_{12}\right)$, similar relations can be derived for $\sigma^{*}$ and $K_{\sigma}$.

Well below the transition temperature, the sixth-order terms in the free-energy expansion also become important. Formally, the Curie-Weiss-type law [Eqs. (1) and (2)] is not valid anymore. In the $a a$ phase, $P_{s}^{2}$ now has a square root dependence on $S_{m}$ so that both linear and square root terms are involved in the explicit expression for $\chi_{33}\left(S_{m}\right)$ given in Ref. 8. In order to evaluate deviations from the CurieWeiss-type law at room temperature, we computed $\chi_{33}\left(S_{m}\right)$ for BT and PT films numerically. ${ }^{9}$

Surprisingly, the dependence $\chi_{33}\left(S_{m}\right)$ was found to be practically linear within the stability range of the $a a$ phase, as shown in Fig. 1 for BT. The plot $\chi_{33}(\sigma)$, computed with the aid of the above relation between $\sigma$ and $S_{m}$, also deviates from the linear one only slightly (Fig. 2). Therefore, the observed variations of the film dielectric response with the misfit strain and internal stress may follow the Curie-Weisstype law [Eqs. (1) and (2)] closely even in the presence of substantial spontaneous polarization. (We recall that, at the

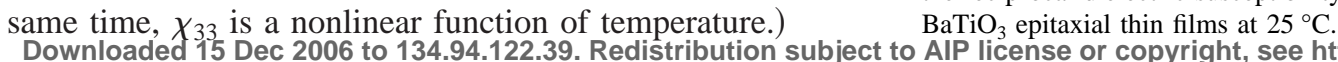

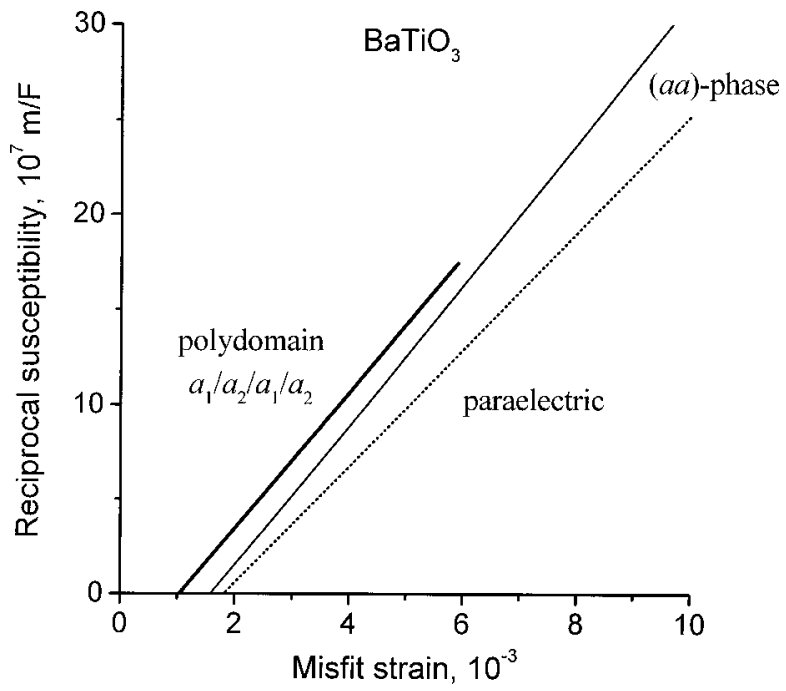

FIG. 1. Misfit-strain dependence of the reciprocal dielectric susceptibility $\chi_{33}$ computed for single domain and polydomain $\mathrm{BaTiO}_{3}$ epitaxial thin films at $25^{\circ} \mathrm{C}$.

To investigate the applicability of Eqs. (1) and (2) further, we have performed similar calculations for polydomain epitaxial thin films. Domain widths were assumed to be much smaller than the film thickness, and the thermodynamic theory of dense laminar domain structures ${ }^{10}$ was used for numerical computations. For BT and PT films grown on tensile substrates, the formation of the so-called $a_{1} / a_{2} / a_{1} / a_{2}$ domain structure ${ }^{11}$ was found to be energetically favorable at the ferroelectric phase transition. ${ }^{12}$ The calculations showed that at room temperature the dependencies $\chi_{33}\left(S_{m}\right)$ and $\chi_{33}(\sigma)$ are almost linear in polydomain BT and PT films as well (see Figs. 1 and 2), which again demonstrate the validity of Eqs. (1) and (2). (It should be emphasized that the domain-wall contribution to the film permittivity equals zero in our case.)

Consider now the parameters involved in the CurieWeiss-type law well below the transition temperature. The critical misfit strain $S_{m}^{*}$, at which the in-plane polarization

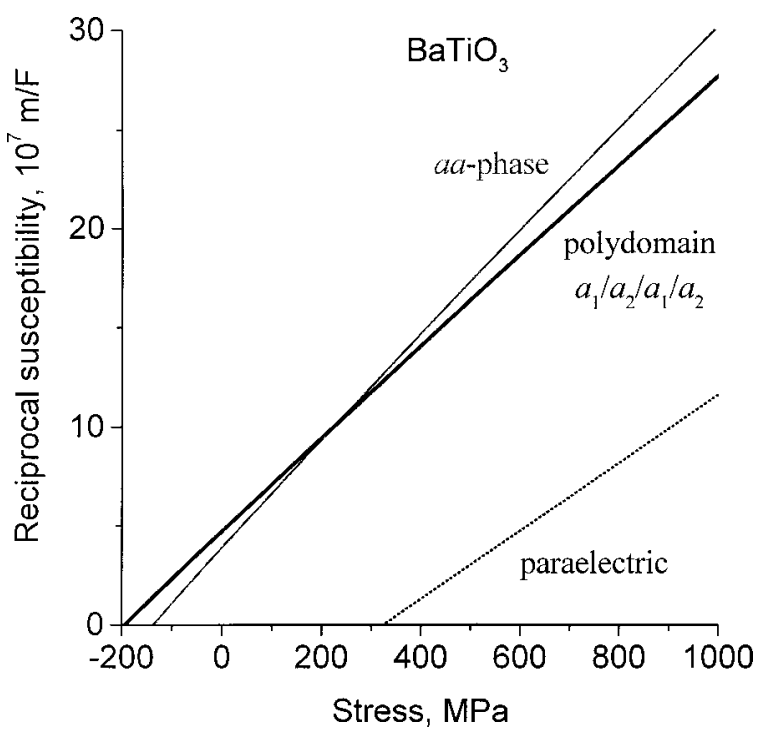

FIG. 2. Theoretical relationships between the in-plane biaxial stress $\sigma$ and the reciprocal dielectric susceptibility $\chi_{33}$ in single domain and polydomain $\mathrm{BaTiO}_{3}$ epitaxial thin films at $25^{\circ} \mathrm{C}$. 
TABLE I. Parameters of the Curie-Weiss-type law for the strain and stress effects on the dielectric response of ferroelectric thin films at $25^{\circ} \mathrm{C}$.

\begin{tabular}{cccccc}
\hline \hline \multirow{2}{*}{ Film } & State & $S_{m}^{*}, 10^{-3}$ & $K_{S}$ & $\sigma^{*}, \mathrm{MPa}$ & $K_{\sigma}, \mathrm{GPa}$ \\
\hline $\mathrm{BaTiO}_{3}$ & $a_{1} / a_{2} / a_{1} / a_{2}$ & 1.03 & 3.16 & -195 & 490 \\
& $a a$ phase & 1.57 & 3.05 & -138 & 428 \\
& paraelectric & 1.80 & 3.68 & 322 & 657 \\
$\mathrm{PbTiO}_{3}$ & $a_{1} / a_{2} / a_{1} / a_{2}$ & -0.76 & 3.27 & -1550 & 448 \\
& $a a$ phase & 9.88 & 6.2 & 126 & 816 \\
& paraelectric & 18.2 & 5.98 & 3310 & 1086 \\
\hline \hline
\end{tabular}

state ( $a a$ or $\left.a_{1} / a_{2} / a_{1} / a_{2}\right)$ loses its stability against the appearance of $P_{3}$, should be calculated from the equality $\chi_{33}$ $=0$, which reduces to a quadratic equation for $S_{m}^{*}$. The critical stress $\sigma^{*}$ is the root of a similar quadratic equation. In turn, $K_{S}$ and $K_{\sigma}$ should be regarded as fitting parameters, which can be extracted from the dependence computed numerically. For BT and PT films at room temperature, the calculated numerical values of $S_{m}^{*}, \sigma^{*}, K_{S}$, and $K_{\sigma}$ are listed in Table I. The values characterizing the prototypic paraelectric state are given here for comparison. It can be seen that the presence of $\mathbf{P}_{\mathbf{s}}$ may strongly influence the parameters of the Curie-Weiss-type law.

We proceed now to the analysis of the experimental data of Shaw et al. on the relationship between the in-plane stress and the dielectric response in $\mathrm{Ba}_{0.7} \mathrm{Sr}_{0.3} \mathrm{TiO}_{3}$ (BST) thin films. ${ }^{7}$ Although the measurements in Ref. 7 were made on polycrystalline films, our approach can be used in this case as well because the straining of a thin film on a thick substrate is a macroscopic phenomenon similar in epitaxial and polycrystalline films. ${ }^{8}$

We first extract the actual permittivity of BST films from the data presented in Ref. 7. The film dielectric constant $\varepsilon_{33}(\sigma)$ may be calculated from the capacitance values $c(\sigma)$ measured in Ref. 7 as $\varepsilon_{33}=t /\left(c^{-1}-c_{i}^{-1}\right)$, where $t$ $=96.4 \mathrm{~nm}$ is the film thickness and $c_{i}$ is the interfacial capacitance caused by the presence of "dead" surface layers with a low permittivity. Taking $c_{i}=0.115 \mathrm{~F} / \mathrm{m}^{2}$ as determined by Streiffer et al. for BST films, ${ }^{13}$ we calculated the pure film permittivity $\varepsilon_{33}$ for various values of $\sigma$ given in Ref. 7. In the absence of external load (residual stress $\sigma$ $=610 \mathrm{MPa}$ ), the relative permittivity was found to be about 570 , which is comparable with other data for $\mathrm{Ba}_{0.7} \mathrm{Sr}_{0.3} \mathrm{TiO}_{3}$ films on platinized Si substrates. ${ }^{13,14}$

For the reciprocal susceptibility $\chi_{33} \approx 1 / \varepsilon_{33}$ plotted versus the in-plane film stress $\sigma$, a linear relationship was obtained. This corresponds to a linear dependence $c(\sigma)$ reported by Shaw et al., ${ }^{7}$ which the authors regarded as a lack of evidence in favor of the presence of spontaneous polarization in the film. According to our results, the linearity of $\chi_{33}(\sigma)$ itself does not indicate the absence of $\mathbf{P}_{\mathbf{s}}$. The experimental value of $K_{\sigma} \approx 1260 \mathrm{GPa}$ has the same order of magnitude as the theoretical value of $K_{\sigma} \approx 500 \mathrm{GPa}$ computed for BT films. Since for BT-based heterostructures the effect of $\mathbf{P}_{\text {s }}$ on $K_{\sigma}$ is relatively weak (see Table I), it is not possible at the moment to gain evidence in favor or against the presence of $\mathbf{P}_{\mathbf{s}}$ from the measured $K_{\sigma}$.

However, the extrapolation of $\chi_{33}(\sigma)$ to zero inverse susceptibility results in a very large effective critical stress $\sigma^{*} \approx-1500 \mathrm{MPa}$. If $\mathbf{P}_{\mathbf{s}}=0$, the critical stress in Eq. (2) may be calculated as $\sigma^{*}=a_{1} /\left(2 Q_{12}\right)=-(T-\theta) K_{\sigma} / C$, where $\theta$ and $C$ are the Curie-Weiss temperature and constant of a bulk material. With $\theta=297 \mathrm{~K}, C=1.18 \times 10^{5} \mathrm{~K}$ (measured for a bulk ceramic ${ }^{15}$ ), and the observed $K_{\sigma}$, we find that $\sigma^{*} \approx-10 \mathrm{MPa}$, which is two orders of magnitude smaller than the experimental estimate. In the presence of $\mathbf{P}_{\mathbf{s}}$, the dependence $\chi_{33}(\sigma)$ may be roughly evaluated for BST films numerically by taking the involved material parameters as weighted averages of those known for pure $\mathrm{BaTiO}_{3}$ and $\mathrm{SrTiO}_{3} .{ }^{4,16}$ The apparent critical stress $\sigma^{*}$, which results from the extrapolation of the part of $\chi_{33}(\sigma)$ located between 600 and $1100 \mathrm{MPa}$, was found to be about $(-200) \mathrm{MPa}$. This value is in better agreement with the experimental one, which in our view indicates the presence of spontaneous polarization in the films in question. ${ }^{17}$

The existence of the in-plane polarization state in BST films grown on Pt-coated Si substrates is further strongly supported by the observed temperature dependence of the permittivity. Indeed, $\varepsilon_{33}(T)$ deviates from the Curie-Weiss law at about $350 \mathrm{~K},{ }^{7,13,14}$ i.e., well above the Curie-Weiss temperature of the bulk material $(297 \mathrm{~K})$. This agrees with the behavior described by the thermodynamic theory, ${ }^{8}$ which predicts the ferroelectric phase transition at about $415 \mathrm{~K}$ in a single crystalline BST film on Si.

Thus, our analysis removes an apparent contradiction between the observed linear variation of the reciprocal susceptibility of BST films with stress and a strong deviation from the Curie-Weiss law in the temperature dependence of the film dielectric response.

${ }^{1}$ N. Setter and R. Waser, Acta Mater. 48, 151 (2000).

${ }^{2}$ Y. Yano, K. Iijima, Y. Daitoh, T. Terashima, Y. Bando, Y. Watanabe, H. Kasatani, and H. Terauchi, J. Appl. Phys. 76, 7833 (1994); T. Kawakubo, S. Komatsu, K. Abe, K. Sano, N. Yanase, and N. Fukushima, Jpn. J. Appl. Phys., Part 1 37, 5108 (1998).

${ }^{3}$ N. A. Pertsev, A. G. Zembilgotov, and A. K. Tagantsev, Phys. Rev. Lett. 80, 1988 (1998).

${ }^{4}$ N. A. Pertsev, A. G. Zembilgotov, and A. K. Tagantsev, Ferroelectrics 223, 79 (1999).

${ }^{5}$ J. S. Speck and W. Pompe, J. Appl. Phys. 76, 466 (1994).

${ }^{6}$ Y. Yoneda, T. Okabe, K. Sakaue, H. Terauchi, H. Kasatani, and K. Deguchi, J. Appl. Phys. 83, 2458 (1998).

${ }^{7}$ T. M. Shaw, Z. Suo, M. Huang, E. Liniger, R. B. Laibowitz, and J. D. Baniecki, Appl. Phys. Lett. 75, 2129 (1999).

${ }^{8}$ N. A. Pertsev, A. G. Zembilgotov, S. Hoffmann, R. Waser, and A. K. Tagantsev, J. Appl. Phys. 85, 1698 (1999).

${ }^{9}$ The sets of BT and PT material parameters listed in Ref. 4 were used in the computations.

${ }^{10}$ N. A. Pertsev and V. G. Koukhar, cond-mat/9907391, (1999); Phys. Rev. Lett. 84, 3722 (2000).

${ }^{11}$ N. A. Pertsev and A. G. Zembilgotov, J. Appl. Phys. 78, 6170 (1995); A. E. Romanov, W. Pompe, and J. S. Speck, ibid. 79, 4037 (1996).

${ }^{12}$ In the $a_{1} / a_{2} / a_{1} / a_{2}$ structure, $90^{\circ}$ domain walls are orthogonal to the film/substrate interface and oriented along the $\{110\}$ planes of the prototypic cubic phase. The spontaneous polarization in the $a_{1}$ and $a_{2}$ domains lies along one of the in-plane edges of the prototypic cubic cell.

${ }^{13}$ S. K. Streiffer, C. Basceri, C. B. Parker, S. E. Lash, and A. I. Kingon, J. Appl. Phys. 86, 4565 (1999).

${ }^{14}$ S. Hoffmann and R. Waser, J. Phys. IV 8, 9 (1998).

${ }^{15}$ A. D. Hilton and B. W. Ricketts, J. Phys. D 29, 1321 (1996).

${ }^{16}$ N. A. Pertsev, A. K. Tagantsev, and N. Setter, Phys. Rev. B 61, R825 (2000).

${ }^{17}$ The fact that $\sigma^{*}$ is still almost one order of magnitude smaller than the experimental estimate may be attributed to the reduction of permittivity and its stress sensitivity in polycrystalline films relative to the single crystalline ones, which is not taken into account in our calculations. 